\title{
Application of disturbance observer-based control on pulsed superconducting radio frequency cavities
}

\author{
Feng Qiu $\odot,{ }^{1,2, * \dagger}$ Takako Miura, ${ }^{1,2}$ Dai Arakawa, ${ }^{1}$ Nao Higashi@ ${ }^{1,2}$ Yosuke Honda® ${ }^{1,2}$ \\ Eiji Kako๑, ${ }^{1,2}$ Toshihiro Matsumoto, ${ }^{1,2}$ Shinichiro Michizono, ${ }^{1,2}$ Tsukasa Miyajima, ${ }^{1,2}$ \\ Takashi Obina, ${ }^{1,2}$ Hiroshi Sakai ${ }^{1,2}{ }^{1,2}$ Kensei Umemori ${ }^{1,2}$ and Masahiro Yamamoto ${ }^{1,2}$ \\ ${ }^{1}$ High Energy Accelerator Research Organization, \\ 1-1 Oho, Tsukuba, Ibaraki 305-0801, Japan \\ ${ }^{2}$ The Graduate University for Advanced Studies, Shonan Village, \\ Hayama, Kanagawa 240-0193, Japan
}

(Received 25 September 2020; accepted 8 January 2021; published 28 January 2021)

\begin{abstract}
A disturbance observer (DOB)-based control approach was verified using the low-level radio-frequency systems of superconducting (SC) cavities operated in a continuous wave (CW) mode in a compact energy recovery linac (cERL) at KEK. This approach compensates for both predictable and unpredictable disturbances in a radio frequency (rf) system such as beam loading, Lorentz force detuning, and microphonics. This paper generalizes the DOB method in pulsed SC rf systems in the cERL at KEK. The results show that the pulse-to-pulse stabilities of the rf system are improved as expected by using this control approach. Furthermore, this method is validated by successfully compensating long pulse beamloading effects in cERL.
\end{abstract}

DOI: 10.1103/PhysRevAccelBeams.24.012804

\section{INTRODUCTION}

At KEK, a compact energy recovery linac (cERL) was constructed in 2013 to demonstrate an energy recovery technique for future $3 \mathrm{GeV}$ ERL-based light sources [1]. The cERL is a $1.3 \mathrm{GHz}$ superconducting radio-frequency (SCRF) test facility operated in continuous wave (CW) mode. A total of six cavities were assembled in the cERL. As shown in Fig. 1, one normal conducting buncher cavity and three two-cell superconducting (SC) cavities (Inj1, Inj2, and Inj3) were installed in the injector; the other two nine-cell SC cavities (ML1 and ML2) were installed in the main linac (ML).

Since 2016, the objective of the cERL facility was changed to cater to superconducting (SC)-technologybased industrial applications [2]. A new beamline was then constructed to produce radioisotopes (RIs) of ${ }^{99} \mathrm{Mo}$ and other medical RIs as shown in Fig. 1 [3]. In addition, we constructed two undulators for building the infrared free electron laser (IR-FEL) beam line in the south section of the recirculation beam line in cERL [4]. Because SC cavities in

\footnotetext{
*qiufeng@impcas.ac.cn

Present address: Institute of Modern Physics, Chinese Academy of Sciences, China.

Published by the American Physical Society under the terms of the Creative Commons Attribution 4.0 International license. Further distribution of this work must maintain attribution to the author(s) and the published article's title, journal citation, and DOI.
}

cERL suffered from degradation caused by thermal breakdown and field emission over the past several years [5-7], the maximum beam energy was decreased to approximately $17.5 \mathrm{MeV}$ during the latest cERL beam commissioning in the $\mathrm{CW}$ mode. However, a higher beam energy is required for further industrial applications related to RI manufacturing and IR-FEL operation. One approach to increase beam energy is operating cERL rf cavities in pulse mode because a higher cavity gradient can be achieved with the pulsed rf. After selecting the appropriate rf pulse patterns and control strategies, we successfully achieved 21.1 MeV beam acceleration with a 7.5-ms pulse duration and a $5-\mathrm{Hz}$ repetition rate [8]. Table I lists the parameters of the SC cavities and their corresponding rf power sources in pulse mode during beam commissioning. Here, $V_{c}, Q_{L}$, and $L_{\text {eff }}$ represent the cavity voltage, cavity loaded Q, and cavity effective length, respectively. Parameter $E$ represents the total beam energy. The beam was operated in pulse mode at $E=17.5 \mathrm{MeV}$ and $E=21.1 \mathrm{MeV}$, as listed in Table I. In the case of $E=17.5 \mathrm{MeV}$, we kept the $V_{c}$ of each cavity the same as that in $\mathrm{CW}$ mode. For $E=21.1 \mathrm{MeV}$, we increased the gradient of cavities in the ML to achieve higher beam energy (Table I). In this paper, we focus on the study of the cavities in the ML.

During the cERL beam commissioning in pulse mode, we observed that fluctuations in cavity detuning were transferred to the rf amplitude and phase; therefore, pulse-to-pulse drifts were observed in the rf field even with feedback (FB) control. These fluctuations were finally 


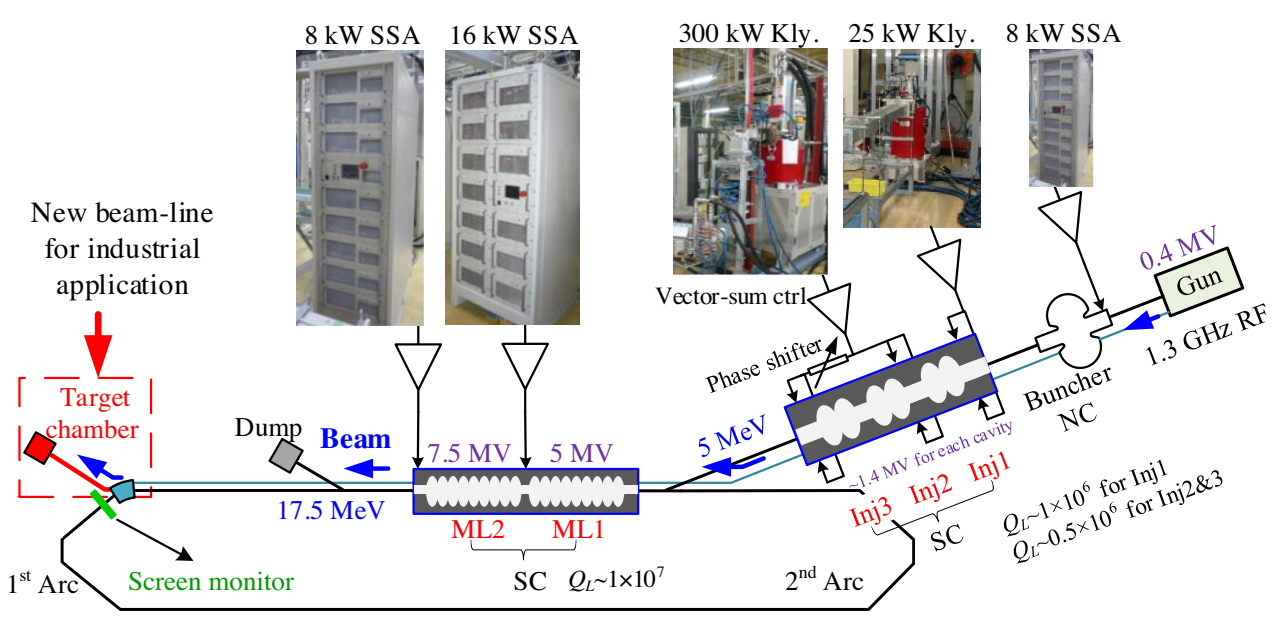

FIG. 1. Layout of cERL. Cavities and rf sources are shown in the figure. The new beamline is indicated by the red block. The marked values of the beam energy and cavity voltage indicates the latest state of the beam commissioning in CW mode. The screen monitor is indicated by the green block.

transmitted to the beam energy based on the measurement results from a screen monitor (Fig. 1) [8]. The currently used proportional integral (PI) FB control could not provide sufficient gain to compensate for beam-loading effects in the case of long pulse beam operation. These issues motivated us to search for other advanced control approaches in addition to the current FB control strategies.

A disturbance observer (DOB)-based control approach was previously applied in cERL to reject various disturbances in the rf system operated in CW mode $[9,10]$. This method was first introduced by Ohnishi and refined by Umeno and Hori [11,12]. The successful DOB control on the $\mathrm{CW}$ rf system inspired us to extend this approach to a pulsed rf system.

The rest of this manuscript is organized as follows. Section II briefly introduces the structure of the pulsed lowlevel radio-frequency (LLRF) system in the cERL and the corresponding control strategies. Section III discusses the limitation of the current control strategies in beam commissioning. Section IV presents the structures of the DOB approach for a pulsed if control system. Then, in Sec. V, we provides the comparison between the performances of our rf system under DOB control and that under PI control. Finally, Sec. VI presents a summary of our study.

\section{LLRF AND TUNER CONTROL}

A schematic of the digital LLRF and tuner FB system is shown in Fig. 2. Two micro telecommunications computer architecture $(\mu \mathrm{TCA})$ field programmable gate array (FPGA) boards (FPGA \#1 and FPGA \#2) are employed in our system. FPGA \#1 board serves as the LLRF field control system, while FPGA \#2 functions as the frequency tuner system.

In the LLRF system, the $1.3 \mathrm{GHz}$ rf signals from the cavity side are down converted to approximately $10 \mathrm{MHz}$ intermediate frequency (IF) signals. The IF signals are sampled at $80 \mathrm{MHz}$ using a 16 bit analog-to-digital converters (ADC), and they are fed to FPGA \#1 to perform digital signal processing [13]. The in-phase and quadrature (I/Q) components of IF signals are extracted [14,15]. After being filtered by a $250 \mathrm{kHz}$ fourth-order infinite impulse response (IIR) filter to attenuate the cavity parasitic mode [16], the I/Q components of the cavity voltage signals are

TABLE I. rf and cavity parameters during the cERL beam commissioning in pulse mode.

\begin{tabular}{lcccrr}
\hline \hline & \multicolumn{3}{c}{$V_{c}$} & & \\
navity & $E=17.5 \mathrm{MeV}$ & $E=21.1 \mathrm{MeV}$ & $Q_{L}$ & $L_{\text {eff }}$ & Power source \\
\hline Inj1 & $1.45 \mathrm{MV}$ & $1.45 \mathrm{MV}$ & $1.2 \times 10^{6}$ & $0.23 \mathrm{~m}$ & $25 \mathrm{~kW} \mathrm{kly.}$ \\
Inj2 & $1.54 \mathrm{MV}$ & $1.54 \mathrm{MV}$ & $0.57 \times 10^{6}$ & & $300 \mathrm{~kW} \mathrm{kly.}$ \\
Inj3 & $1.30 \mathrm{MV}$ & $1.30 \mathrm{MV}$ & $0.48 \times 10^{6}$ & & $16 \mathrm{~kW}$ SSA \\
ML1 & $5 \mathrm{MV}$ & $5.5 \mathrm{MV}$ & $1.3 \times 10^{7}$ & $1.036 \mathrm{~m}$ & $8 \mathrm{~kW}$ SSA \\
ML2 & $7.5 \mathrm{MV}$ & $10.5 \mathrm{MV}$ & $1.1 \times 10^{7}$ & & \\
\hline \hline
\end{tabular}




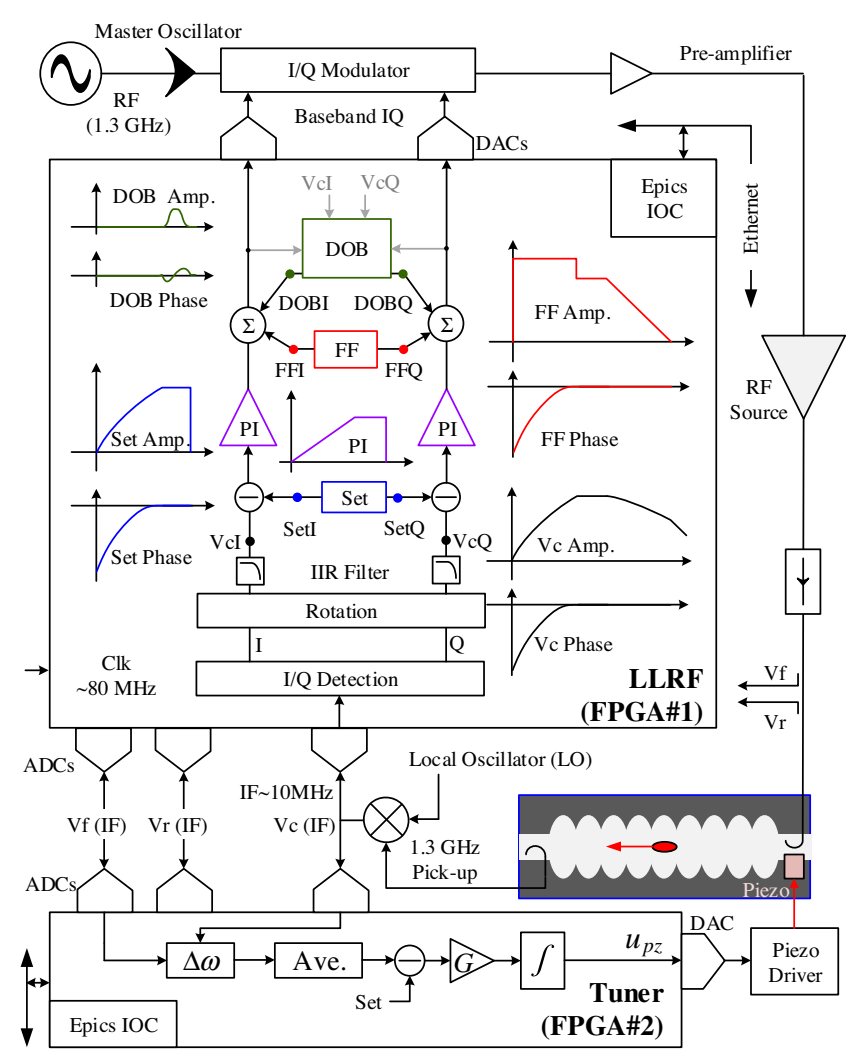

FIG. 2. Schematic of the digital LLRF and tuner system in the cERL. The pulse patterns for the cavities in the main linac are illustrated in the figure for better explanation.

compared with the set table. The I/Q errors are calculated and regulated using a PI FB controller. The regulated I/Q signals are added to a static feedforward (FF) table (the output of the DOB controller is added in the case of PI + DOB control). The combined signals are fed to the I/Q modulator to modulate the rf signal from the master oscillator. Finally, the modulated rf signal are fed to a high power rf source, which drives the cavities. In Fig. 2, the pulse patterns of the FF table, set table, cavity voltage signals, and DOB controller output are illustrated for better understanding.

In the tuner system (FPGA \#2), the down conversion and IQ detection part are the same as those in the LLRF system. Cavity detuning $(\Delta \omega)$ during the rf pulse can be calibrated using [17]

$$
\Delta \omega=\frac{d \phi}{d t}-\frac{\omega_{1 / 2}\left(\left|2 \vec{V}_{f}\right|\right) \sin (\theta-\phi)}{\left|\vec{V}_{c}\right|},
$$

where $\phi$ and $\theta$ represent the phases of $V_{c}$ and $V_{f}$, respectively. The quantity $\omega_{1 / 2}$ is the cavity half bandwidth. The averaged detuning error is regulated with gain $G$, and then, it is added to the piezo control signal $u_{p z}$ from the last pulse, a pulse-to-pulse FB mechanism is established based on this method [8].

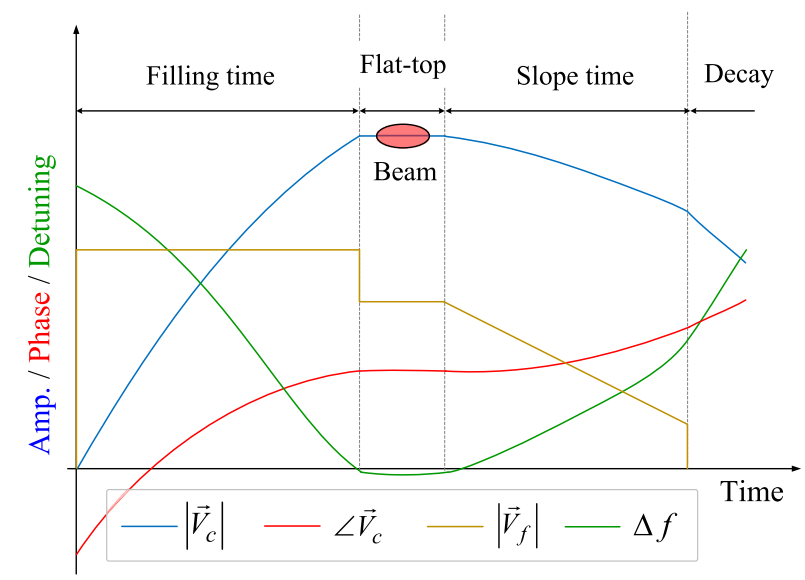

FIG. 3. Typical amplitude and phase pulse pattern of the cavity voltage $\left(V_{c}\right)$, amplitude pattern of the cavity forward voltage $\left(V_{f}\right)$, and the corresponding lorentz force detuning (LFD) during the cERL beam commissioning.

Figure 3 illustrates a typical rf pulse pattern and the corresponding lorentz force detuning (LFD) in pulse mode. The $V_{f}$ in the figure indicates the cavity forward voltage. The filling time represents the stage of filling the rf voltage to the designed value. Owing to the limited capabilities of the power sources in the ML cavities and high $Q_{L}$ of more than $1.0 \times 10^{7}$, a long filling time up to $3.5 \mathrm{~ms}$ was adopted for ML1 and ML2 in the early commissioning [8]. In the latest beam commissioning, the filling time was extended to $5 \mathrm{~ms}$ to further decrease the required incident power during the filling time. Following the filling time, a $1 \mathrm{~ms}$ flat-top was maintained for beam acceleration. To satisfy the power limits of the cavity coupler before switching off the rf, a $4 \mathrm{~ms}$ (or $3 \mathrm{~ms}$ for early commissioning) "slope time" is inserted to reduce the transient reflected power [8].

During the filling time of the rf pulse, we should focus on a large LFD proportional to the square of the accelerating gradient [18]. For ML2 operated with $10.5 \mathrm{MV} V_{c}$, the maximum LFD is about $85 \mathrm{~Hz}$; it is roughly 1.5 times the cavity half-bandwidth $(\approx 57 \mathrm{~Hz})$. Figure 4 shows the detuning of the ML2 ( $\left.V_{c}=10.5 \mathrm{MV}\right)$ calibrated using (1). Because we applied a long filling time of up to $3.5 \mathrm{~ms}$,

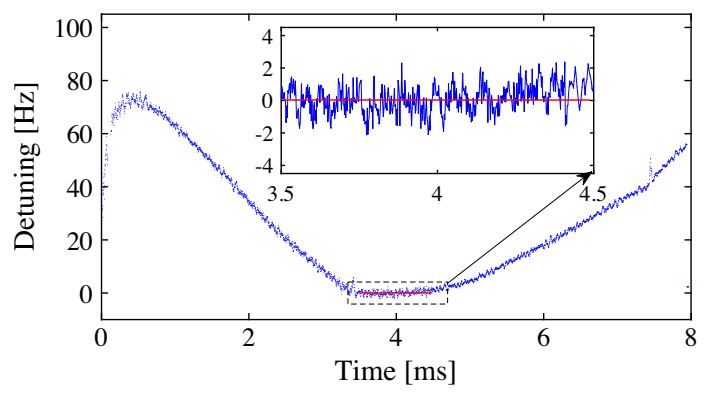

FIG. 4. Examples of cavity detuning on ML2 that operated at 10.5 MV in the pulse operation. The red line indicates the mean value of the detuning during the flat-top. 

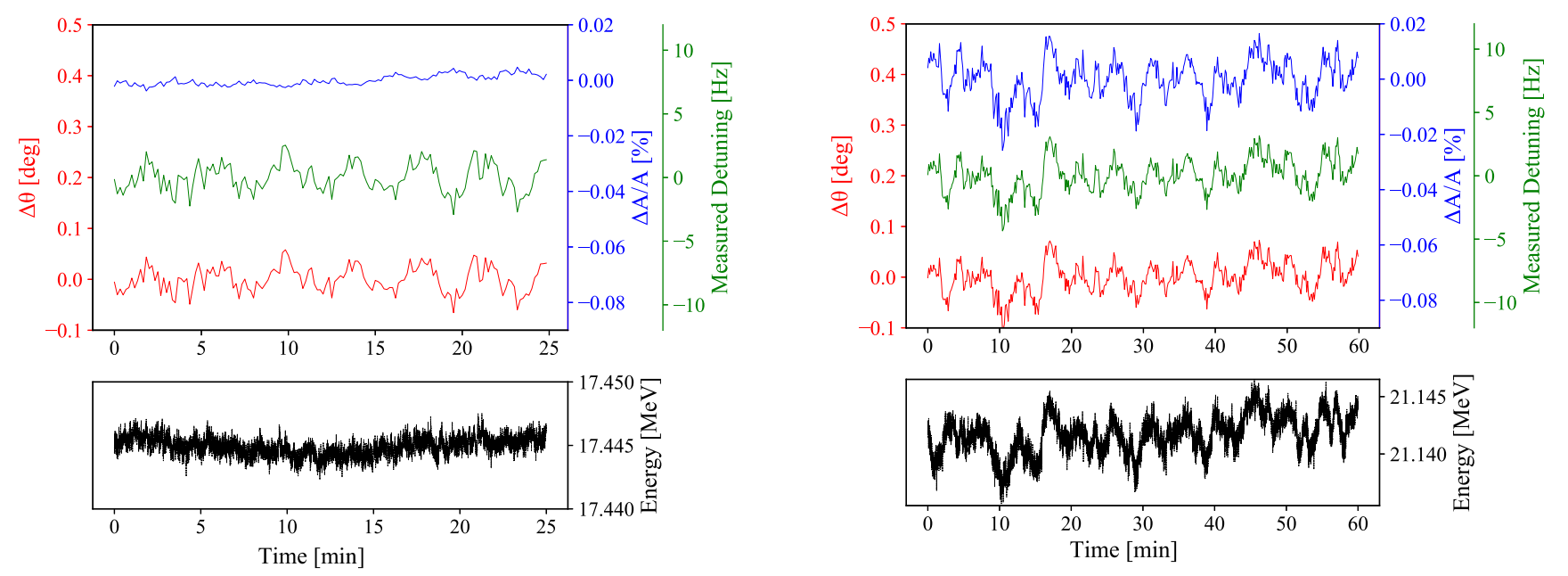

FIG. 5. Long term pulse-to-pulse amplitude (blue), phase (red) and detuning (green) during the flat-top of the rf pulse on ML2 that operated at $7.5 \mathrm{MV}$.

the LFD almost reaches its steady state at the beginning of the flat-top. That is, the LFD during the $1 \mathrm{~ms}$ flat top is almost constant as shown in Fig. 4. Thus, the compensation of the intra-pulse detuning is not considered in this paper. Based on (1), we can calibrate the average value of the detuning red line in Fig. 4) during the flat-top in an rf pulse (in the case w/o beam or with short pulse beam).

In addition, a filling on resonance method is adopted to reduce incident power during the filling time $[19,20]$. The phase of the FF table is modulated to track the cavity resonance frequency as shown in Fig. 2. These pulse patterns and control strategies are discussed in detail in Ref. [8].

We commissioned the current LLRF and tuner system in the cERL beam commissioning in pulse mode. Figure 5 shows the result of a $17.5 \mathrm{MeV}$ beam acceleration with a pulsed rf. The $0.45 \mathrm{~mA}$ beam with a pulse duration of $0.9 \mu \mathrm{s}$ and a $5 \mathrm{~Hz}$ repetition rate was accelerated on-crest. The beam-loading effects are almost negligible because of the short beam pulse duration. The beam energy is calibrated based on the peak point of the beam projection on the screen monitor (Fig. 1). For comparison, the pulseto-pulse detuning (green), $V_{c}$ amplitude (blue), and $V_{c}$ phase (red) during the flat-top of the rf pulse for ML2 was plotted as well. The cavity is operated at $7.5 \mathrm{MV}$ as listed in Table I. According to Fig. 5, the drifts in the detuning caused by the pressure fluctuation is coupled to the rf phase rather than the rf amplitude. Fortunately, the beam energy is not affected by the rf phase compared with the rf amplitude because of the on-crest acceleration.

\section{LIMITATION OF CURRENT CONTROL STRATEGY}

We have to increase the $V_{c}$ of ML2 to $10.5 \mathrm{MV}$ to realize a $21.1 \mathrm{MeV}$ beam acceleration. Figure 6 compares the measured beam energy, pulse-to-pulse detuning, and the

FIG. 6. Long term pulse-to-pulse amplitude (blue), phase (red) and detuning (green) during the flat-top of the rf pulse on ML2 at 10.5 MV. An obvious beam energy dirft (black) was observed.

corresponding rf amplitude and phase. Unlike the case of the $17.5 \mathrm{MeV}$ beam acceleration, the drifts in the detuning was coupled to not only the rf phase but also the rf amplitude. These drifts were transferred to the beam, and led to an obvious beam energy drift.

In practice, after calculating the dynamic detuning with (1), the tuner control system regulates the piezo tuner to ensure that the measured average detuning in the flat-top always fluctuates around $0 \mathrm{~Hz}$. However, a detuning offset $\left(\Delta f_{\text {offset }}\right)$ may exist between the actual and measured detuning.

Figure 7 shows the cavity resonance curves for amplitude and phase as a function of detuning $\Delta \omega\left(\Delta \omega=\omega_{0}-\omega\right)$, where parameters $\omega_{0}$ and $\omega$ represent the cavity resonance frequency and $\mathrm{rf}$ frequency, respectively. As shown in Fig. 7(a), the $V_{c}$ amplitude is less sensitive to the detuning drift compared with the $V_{c}$ phase as long as the cavity is operated on resonance $\left(\Delta f_{\text {offset }}=0\right)$. However, as shown in Fig. 7(b), the existence of the $\Delta f_{\text {offset }}$ can led to a larger amplitude fluctuation.

If the rf system is operated in the open-loop mode, it means the static FF table directly drives the rf source without responding to how the cavity reacts. The phase and amplitude drifts $\left(\Delta \theta_{\mathrm{ol}}, \Delta A_{\mathrm{ol}}\right)$ caused by the detuning drift $\Delta \omega_{\text {drift }}$ can be expressed as [14]

$$
\left\{\begin{array}{l}
\Delta \theta_{\mathrm{ol}}=\arctan \frac{\Delta \omega_{\mathrm{drift}}}{\omega_{0.5}}, \\
\Delta A_{\mathrm{ol}}=\frac{1}{\sqrt{1+\tan ^{2}\left(\Delta \theta_{\mathrm{ol}}\right)}},
\end{array}\right.
$$

Eq. (2) is only available in the case of static detuning. In our case, as mentioned above, the LFD almost reaches its steady state during the flat-top (Fig. 4). Therefore, we consider the detuning caused by the slow drifts as a static detuning. 


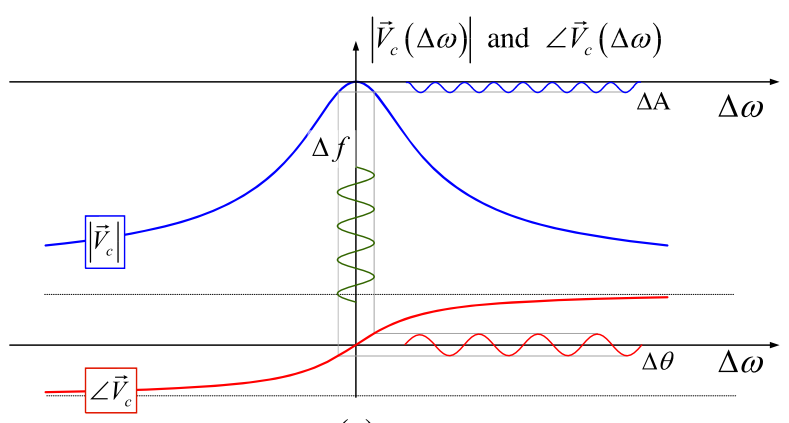

(a)

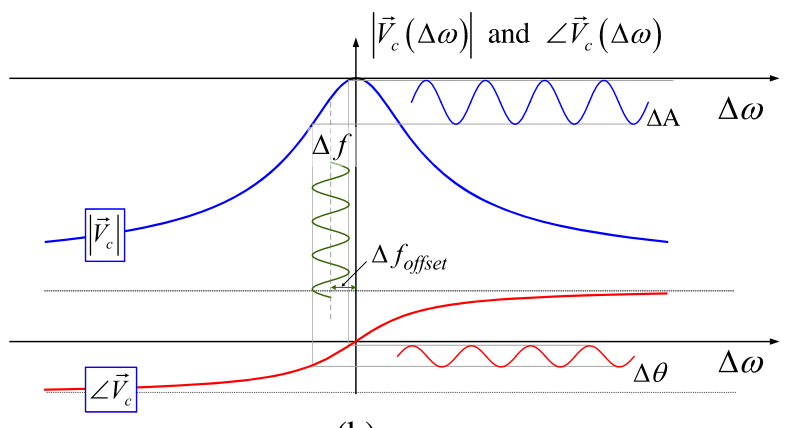

(b)

FIG. 7. Resonance curves for the amplitude and phase of a cavity as a function of detuning $\left(\Delta \omega=\omega_{0}-\omega\right)$ in the case of (a): $\Delta f_{\text {offset }}=0$ and (b): $\Delta f_{\text {offset }}<0$.

In the closed-loop mode, drifts are attenuated by the FB gain $G_{f b}$; thus, we have

$$
\left\{\begin{array}{l}
\Delta \theta_{F B}=\frac{\Delta \theta_{\mathrm{ol}}}{1+G_{f b}}, \\
\Delta A_{F B}=\frac{\Delta A_{\mathrm{ol}}}{1+G_{f b}},
\end{array}\right.
$$

where $\Delta \theta_{F B}$ and $\Delta A_{F B}$ represent the drifts of the phase and amplitude under FB control, respectively. Figure 8 shows the calibrated drifts based on (5) and (8). The upper plot shows that the case of $\Delta f_{\text {offset }}$ is not included in the actual detuning. The amplitude drift was not observed as expected (this is also the case of $V_{c}=7.5 \mathrm{MV}$, as shown in Fig. 5).

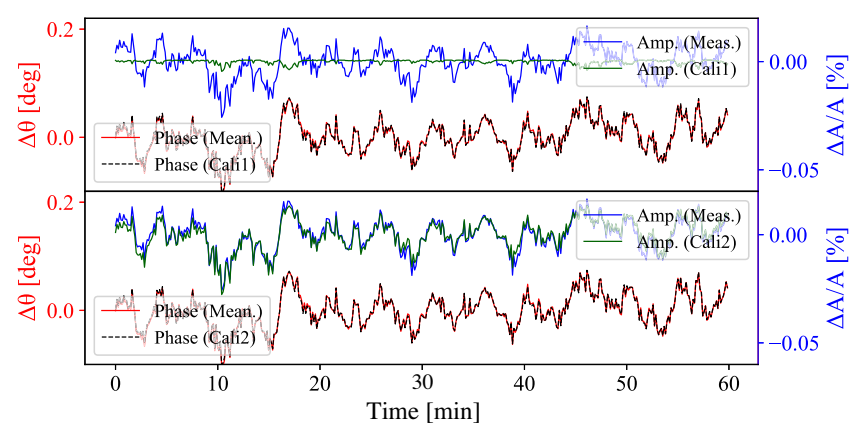

FIG. 8. Comparison of calibrated amplitude (and phase) drift and measured amplitude (and phase) on cavity ML2 that operated at $10.5 \mathrm{MV}$. From top to bottom: $\Delta f_{\text {offset }}=0 \mathrm{~Hz}$, and $\Delta f_{\text {offset }}=-8 \mathrm{~Hz}$.
The lower plots assume that there is a $-8 \mathrm{~Hz} \Delta f_{\text {offset }}$ in the actual detuning. The calibrated and measured amplitude drifts are in good agreement. This is because the $-8 \mathrm{~Hz}$ $\Delta f_{\text {offset }}$ is not well-understood so far, and it is noted that this $\Delta f_{\text {offset }}$ is not observed in ML2 at 7.5 MV. A possible reason for this $\Delta f_{\text {offset }}$ is the calibration error in (1) caused by the limited directivity of the rf couplers. The existence of the $\Delta f_{\text {offset }}$ when operating the cavity with a higher gradient limits the pulse-to-pulse stability. The current PI FB control is not sufficient to reject this detuning drifts as shown in Fig. 6.

Another limitation of the current control approach is the compensation of the long pulse beam loading. In principle, beam loading can be compensated using high FB gains. However, high FB gains not only increase the risk of an unstable system but also increase the noise level of the high frequency components. To optimize FB gains, a gainscanning experiment was performed in the absence of the beam. Optimal gains were determined based on the scanned performance curves. In this paper, the PI controller is defined by its continuous form

$$
C_{\mathrm{PI}}(s)=K_{P}+\frac{K_{I}}{s},
$$

where $K_{P}$ and $K_{I}$ represent the value of the proportional gain and integral gain, respectively. Under the optimal integral gain $K_{I}$ (approximately $1.2 \times 10^{5}$ in our case), amplitude and phase stabilities as a function of $K_{P}$ are shown in Fig. 9. First, we determined the optimal gain based on its phase performance for ML1 and ML2.
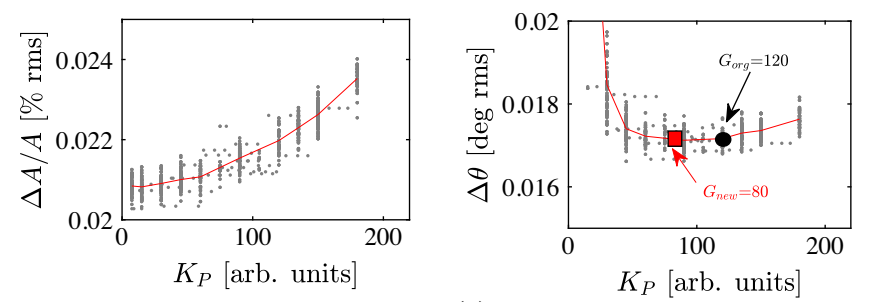

(a)
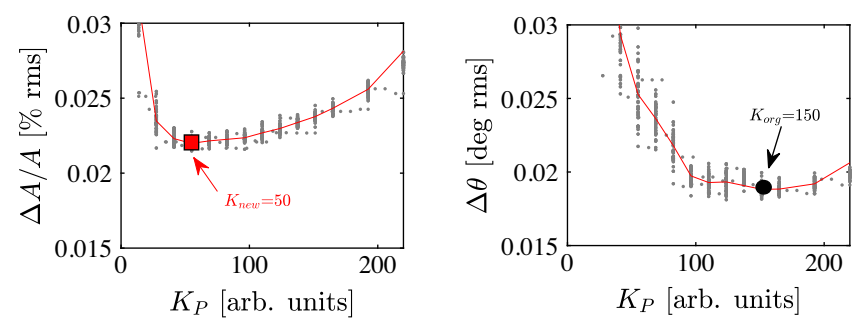

(b)

FIG. 9. Gain-scanning result for (a) ML1, (b) ML2. The subplots on the left and right indicate the amplitude and phase stabilities, respectively. Before $2014, G_{\text {org }}$ and $K_{\text {org }}$ were determined as the optimal gains based on the phase performance. From 2015 to the present, $G_{\text {new }}$ and $K_{\text {new }}$ were selected as the operational gain to reduce the rf trips. 

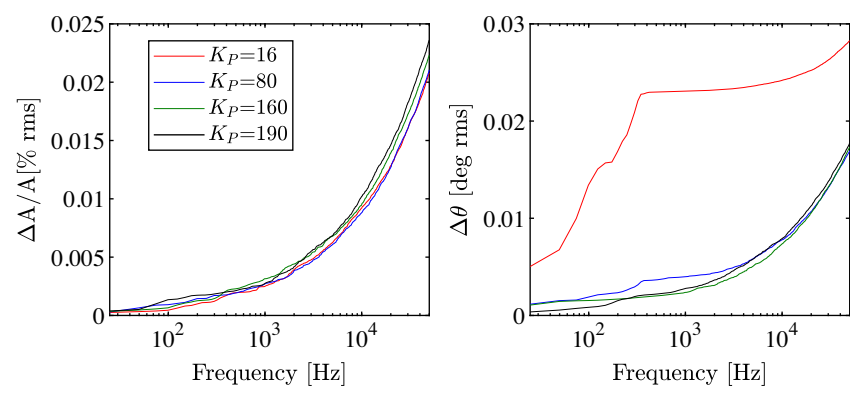

FIG. 10. Cumulative RMS amplitude (left) and phase (right) stabilities of ML1 under different $K_{P}$.

However, rf trips occurred in ML1 and ML2 because of the high FB gains. These scenarios forced us to decrease the gain from $G_{\text {org }}$ and $K_{\text {org }}$ to $G_{\text {new }}$ and $K_{\text {new }}$ after the year 2015, respectively. The gain $K_{\text {new }}$ is selected as the operational gain of ML2 on the basis of amplitude performance (optimal amplitude stability). The gain $G_{\text {new }}$ was determined for the operational gain of ML1 to compromise between amplitude and phase stabilities. The rf trips caused by the LLRF system were significantly reduced as presented in Ref. [21].

Figure 10 compares the cumulative RMS stability of ML1 with different $K_{P}$ but with the same $K_{I}$ $\left(K_{I}=1.2 \times 10^{5}\right)$ in CW mode. From the amplitude and phase cumulative RMS stability curves, it can be seen that higher gains lead to a deteriorated stability in the rf field owing to the enhancement of high frequency noise. Based on what we have mentioned above, in order to improve both of the robustness and performance of the system, we

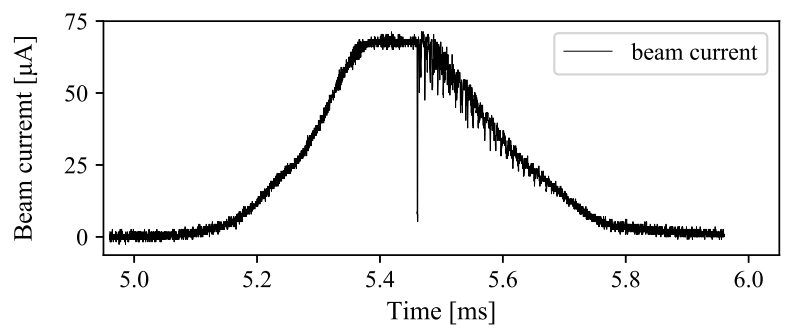

(a)

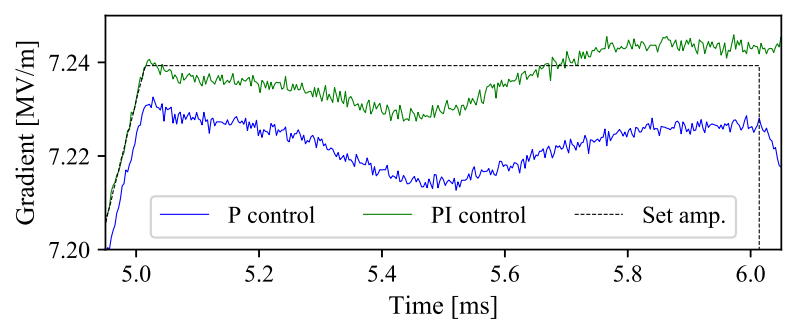

(b)

FIG. 11. (a) The $0.7 \mathrm{~ms}$ and $66 \mu \mathrm{A}$ peak current beam measured by the oscilloscope. (b) Measured rf field for the amplitude on ML2 in the case of P control (blue) and PI control (green). have selected $K_{P}=80$ and $K_{P}=50$ for ML1 and ML2, respectively.

Figure 11(a) shows the $0.7 \mathrm{~ms}$ and $66 \mu \mathrm{A}$ beam current used in beam commissioning. Since the cavity was operated in the on-crest mode, the beam-loading effects were concentrated on the rf amplitude. Figure 11(b) shows the amplitude result at the flat-top for the P control and PI control on ML2 $\left(V_{c}=7.5 \mathrm{MV}\right)$. The beam-induced peakto-peak amplitude error for both cases are approximately $0.2 \%$. The FB gains in the experiments are $K_{P}=50$ and $K_{I}=1.2 \times 10^{5}$. To further improve system performance, DOB control approaches we successfully applied in the $\mathrm{CW}$ rf system were considered.

\section{DOB CONTROL}

In the cERL beam commissioning of the $\mathrm{CW}$ SC cavities, the DOB control method was demonstrated by successfully compensating for beam loading, and by the suppression of the power supply ripples and microphonics [9]. The key component of DOB control is a disturbance observer, which can estimate the disturbances of the system. The structure of the DOB control is illustrated in Fig. 12(a). Signals $u, d$, and $\hat{d}$ represent plant input, disturbance, and disturbance estimate, respectively. In principle, the signal $d$ represents the disturbances from

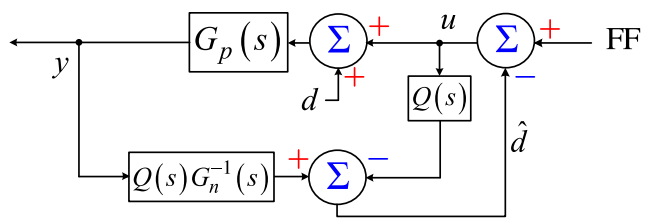

(a)

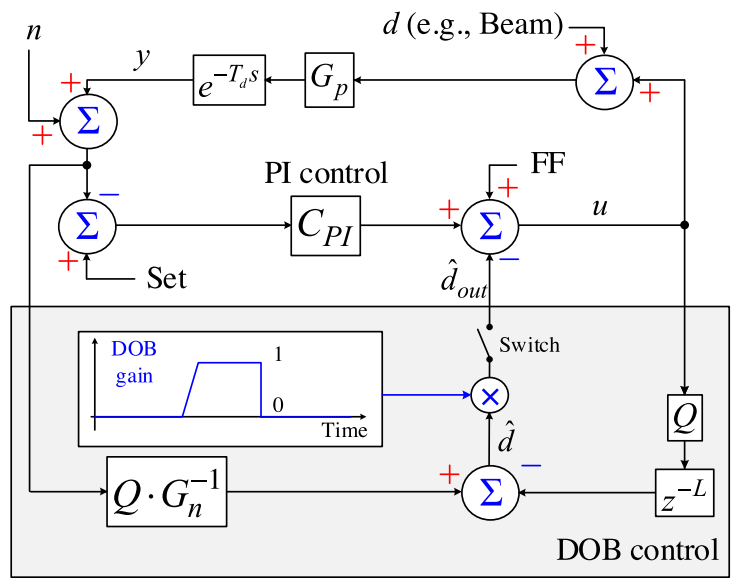

(b)

FIG. 12. (a): Basic structure of DOB control. The disturbance signal $d$ can be estimated by the DOB controller output $\hat{d}$. The effective bandwidth of the controller is determined by the lowpass $Q$ filter. (b): Simplified architecture of DOB control in combination with PI control. 
the plant input, such as the beam loading and power supply ripples. However, the DOB control has proven to be effective in successfully rejecting the microphonics detuning which does not directly affect the plant input [9]. One possible reason is that the detuning error can be equivalent to the phase error of the cavity input if the cavity is operated approximately on resonance. Therefore, such detuning errors can also be dealt with by the DOB controller.

Models $G_{p}$ and $G_{n}$ represent the transfer function of the real plant (combination of cavity, rf source, preampifier, IQ detector, etc.) and nominal system models, respectively. Generally, the model $G_{n}$ can be obtained by modern system identification [22]. In our case, because the cavity half bandwidth $(\approx 50 \mathrm{~Hz})$ is much smaller than other components, the dominant poles of $G_{p}$ are contributed by the cavity itself. Furthermore, if the cavity detuning, $\Delta f$, is well compensated by the tuner system, and the phase calibration error $\Delta \theta$ is negligible. The transfer function of $G_{n}(s)$ under these ideal conditions can be simplified by

$$
G_{n}(s)=\frac{G_{f f} \cdot 2 \pi f_{0.5}}{s+2 \pi f_{0.5}},
$$

where the parameter $G_{f f}$ and $f_{0.5}$ represent the plant gain and cavity half bandwidth, respectively.

The filter $Q(s)$ is used to make the $G_{n}^{-1}$ physically realizable [12]. In our design, the $Q$ filter is a second-order low-pass filter with the transfer function [9]

$$
Q(s)=\frac{1}{(\tau s+1)^{2}},
$$

where $\tau$ is related to the filter bandwidth.

According to Fig. 12, the disturbance estimation is given by $[9,10]$

$$
\hat{d}=Q\left[(u+d) G_{p} G_{n}^{-1}-u\right] .
$$

If the system model has high accuracy $\left(G_{n} \approx G_{p}\right)$, it can be seen that $\hat{d} \approx Q \cdot d$. If the $Q$ filter is a low-pass filter with gain 1 as shown in (6), $\hat{d} \approx d$ in the low-frequency domain; therefore, we can use this disturbance estimate $\hat{d}$ to cancel the real disturbance (in the low-frequency domain), as shown in Fig. 12(a).

We incorporated the DOB control with the PI controller. The overall control block diagram is shown in Fig. 12(b). The signal $n$ represents the measurement noise in the system. The model $C_{\mathrm{PI}}$ represents the PI controller and the DOB controller is indicated by the grey block. The actual rf system has time delay, $T_{d}$, and it should be compensated by the digital delay unit $G_{T_{n}}$ :

$$
G_{T_{n}}=z^{-L}, \quad L=\left\lfloor\frac{T_{d}}{T_{s}}\right\rfloor,
$$

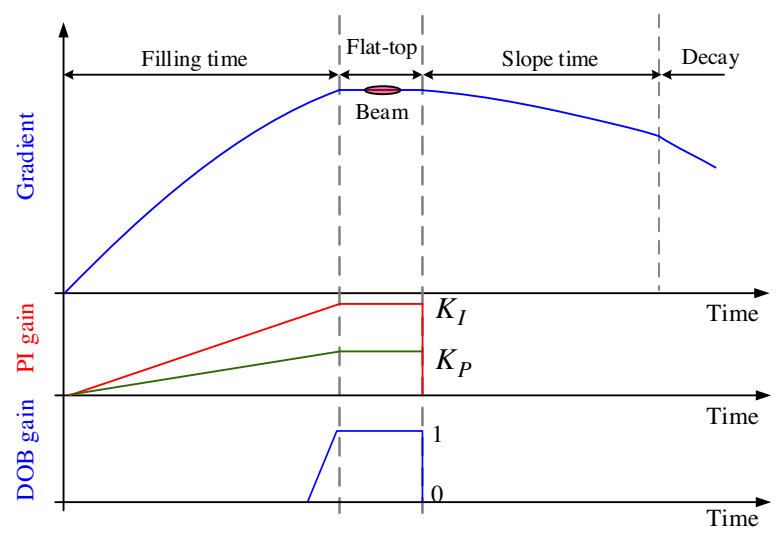

FIG. 13. Activation time of each PI control and DOB control.

where the operator " $\rfloor\rfloor$ " means round the element inside the brackets to the nearest integers toward 0 , and the parameter $T_{s}$ represents the sampling period of the FPGA board.

To achieve better transient performance, the two controllers were activated with different timelines as shown in Fig. 13. The PI gain gradually increased from 0 (at the beginning of the filling time) to the steady-state value (at the beginning of the flat-top) and it remained constant in the flat-top. The DOB control was activated in the latter part of the filling time, and in the entire flat-top. In Fig. 12(b), the disturbance estimate $\hat{d}$ is modulated with the DOB gain, and it yields an actual controller output $\hat{d}_{\text {out }}$.

During the flat-top (steady-state) of the rf pulse, an analytical study of the PI + DOB control was carried out. Since there are three internal loops in Fig. 12, we have adopted the Mason's rule to analyze the system [23]. The characteristic polynomial $\Delta_{t o l}$ (system determinant) in the overall schematic is defined by

$\Delta_{\mathrm{tol}}=1-(\underbrace{Q \cdot G_{T_{n}}}_{L_{1}} \underbrace{-Q G_{n}^{-1} \cdot G_{p} e^{-T_{d} s}}_{L_{2}} \underbrace{-C_{\mathrm{PI}} \cdot G_{p} e^{-T_{d} s}}_{L_{3}})$,

where $L_{1}, L_{2}$ and $L_{3}$ represent the three internal loops in Fig. 12. If we operate the system with PI individual control, the characteristic polynomial $\Delta_{\mathrm{PI}}$ becomes

$$
\Delta_{\mathrm{PI}}=1-(\underbrace{-C_{\mathrm{PI}} \cdot G_{p} e^{-T_{d} s}}_{L_{3}}) .
$$

As shown in (9), if the nominal model $G_{n}$ is exactly same with $G_{p}$, and the loop delay $e^{-T_{d} s}$ is perfectly compensated by the digital delay $G_{T_{n}}$, the internal loops $L_{1}$ and $L_{2}$ cancel out. With these conditions, we have $\Delta_{\text {tol }}=\Delta_{\mathrm{PI}}$. Here we suppose the cavity is operated on resonance and there is no phase calibration error. 
TABLE II. Assumptions in the analytical study.

\begin{tabular}{lrl}
\hline \hline Item & Value & \multicolumn{1}{c}{ Definition } \\
\hline$\frac{\delta G_{f f}}{G_{f f}}$ & $10 \%$ & Mismatch of plant gain \\
$\frac{\delta f_{0.5}}{f_{0.5}}$ & $15 \%$ & Mismatch of cavity half bandwidth \\
$\frac{\Delta L}{L}$ & $50 \%$ & Mismatch of loop delay \\
$\Delta f_{\text {offset }}$ & $-20 \mathrm{~Hz}$ & Detuning offset \\
$\Delta \theta$ & $5 \mathrm{deg}$ & Phase calibration error \\
\hline \hline
\end{tabular}

In practice, a mismatch always exists between $G_{p}$ and $G_{n}$. Because the two dominant parameters in $G_{n}$ are plant gain $G_{f f}$ and cavity bandwidth $f_{0.5}$, we inspected these two parameters. We suppose the mismatched $G_{n}$ is expressed as

$$
G_{n}(s)=\left(G_{f f}+\delta G_{f f}\right) \cdot \frac{2 \pi\left(f_{0.5}+\delta f_{0.5}\right)}{s+2 \pi\left(f_{0.5}+\delta f_{0.5}\right)} .
$$

Similarity, we assume that there is a deviation in the estimation of loop delay:

$$
G_{T_{n}}=z^{-(L+\Delta L)}
$$

In addition, to make the analytical study more realistic, we suppose that the small detuning error and the phase calibration error cannot be neglected in the actual plant. The $G_{p}$ is then given by

$$
G_{p}(s)=G_{f f} \cdot \frac{2 \pi f_{0.5}}{s+2 \pi\left(f_{0.5}-j \cdot \Delta f_{\text {offset }}\right)} \cdot e^{-j \Delta \theta} .
$$

Table II summarize the assumptions and restrictions we have used in the analytical study. In general, these restrictions can be satisfied in the cERL. Next, from Fig. 14 to Fig. 18, we use the same assumptions described in Table II.

Figure 14 compares the bode plots of the sum of all loop gains (i.e., $-1+\Delta_{\text {tol }}$ and $-1+\Delta_{\mathrm{PI}}$ ) in the case of the PI + DOB control and PI individual control on ML1. The cutoff bandwidth ( $3 \mathrm{~dB}$ bandwidth) of the $Q$ filter $f_{Q}$ is set to $5 \mathrm{kHz}$. The loop delay is approximately $1 \mu \mathrm{s}$. The PI gains here are 80 and $1.2 \times 10^{5}$, respectively.

According to Fig. 14, the bode plots in the case of the with and without DOB control almost overlap. The maximum extra phase lag caused by DOB controller is approximately 6 degrees, which means the introduction of the DOB structure will not significantly influence the total characteristic polynomial of the system.

The improvement of PI + DOB control can be explained by inspecting its closed-loop transfer function from $d$ to $y$. According to Mason's rule, the transfer function of PI + $\mathrm{DOB}$ is given by
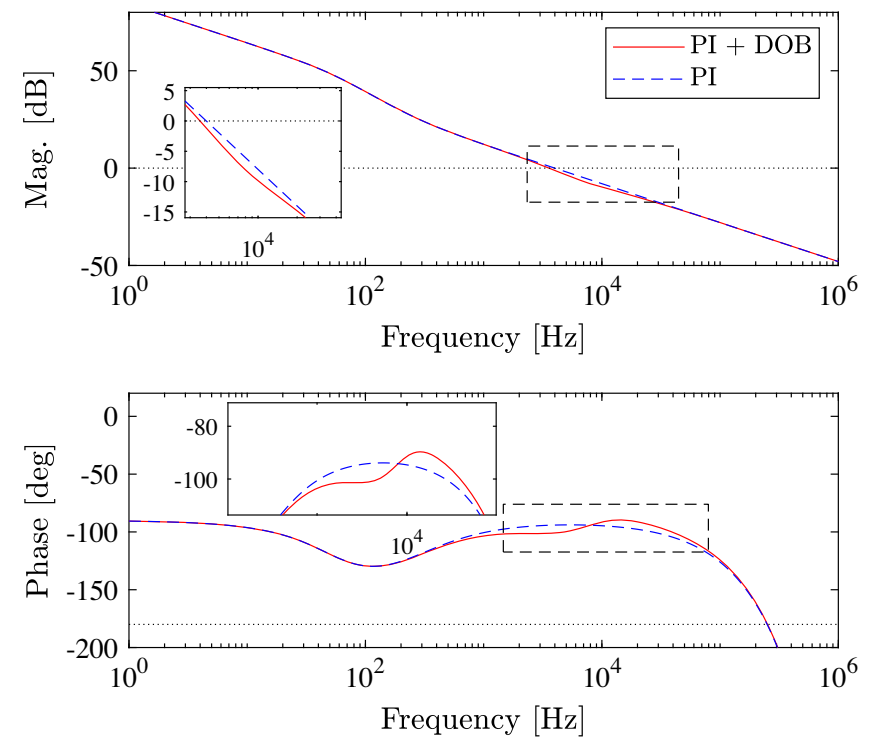

FIG. 14. Comparison of the bode plots of the sum of all loop gains in the case of the PI + DOB control and PI individual control. The magnitude response in the two cases almost overlap. The maximum phase lag caused by DOB control is approximately 6 degrees.

$$
H_{\mathrm{tol}, d \rightarrow y}=\left(1-Q \cdot G_{T_{n}}\right) \cdot \frac{G_{p} \cdot e^{-T_{d} s}}{\Delta_{t o l}} .
$$

On the other hand, the transfer function of PI + DOB is given by

$$
H_{\mathrm{PI}, d \rightarrow y}=\frac{G_{p} \cdot e^{-T_{d} s}}{\Delta_{\mathrm{PI}}}
$$

As shown in Fig. 14, the denominator of (14) and (15) is almost same, the main difference of these two transfer functions is $1-Q \cdot G_{T_{n}}$ with the magnitude response as shown in Fig. 15. According to Fig. 15, the factor $1-Q$. $G_{T_{n}}$ will improve the system response from $d$ to $y$ in the frequencies ranging from direct current (DC) to $f_{Q}$.

Figure 16 plots the closed-loop transfer function from the disturbance signal $d$ to the cavity pickup signal $y$. Here, we assume that the model mismatches and the detuning and phase errors are given in Table II. The results indicate that the presented DOB structure shows better performance in terms of disturbance rejection for the low-frequency range.

In DOB control, the bandwidth of the $Q$ filter restricts the effective bandwidth of the controller. The DOB control does not work if the frequency of the disturbance is higher than $f_{Q}$. Figure 17 shows the upper boundary for the singular value of the closed loop transfer function from $d$ and $n$ to $y$ in terms of different $f_{Q}$ under P + DOB control. The determination of $f_{Q}$ is a compromise between disturbance rejection and noise suppression according to Fig. 17. 


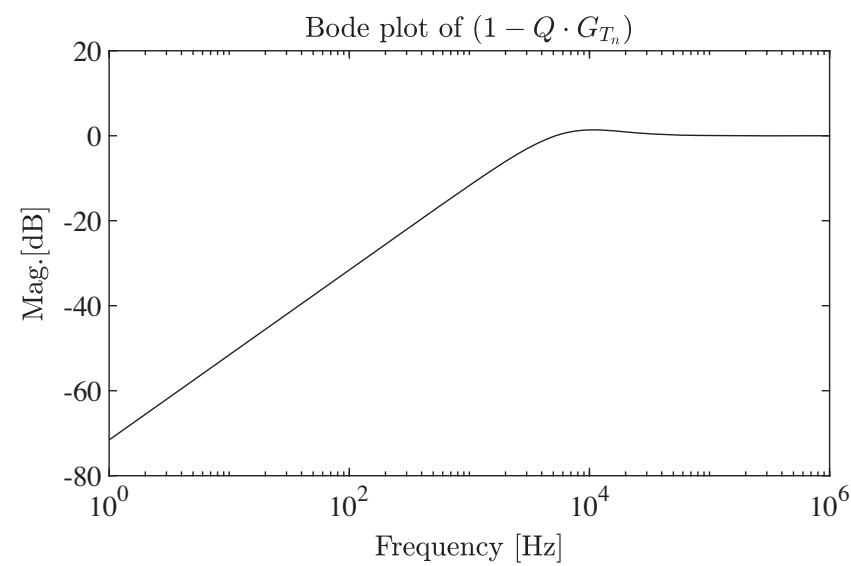

FIG. 15. Bode plot of the block, $1-Q \cdot G_{T_{n}}$. In the frequency range from DC to $f_{Q}$, we have $\left|1-Q \cdot G_{T_{n}}\right|<1$. As a result, the closed-loop system response from $d$ to $y$ will be improved by the DOB control.

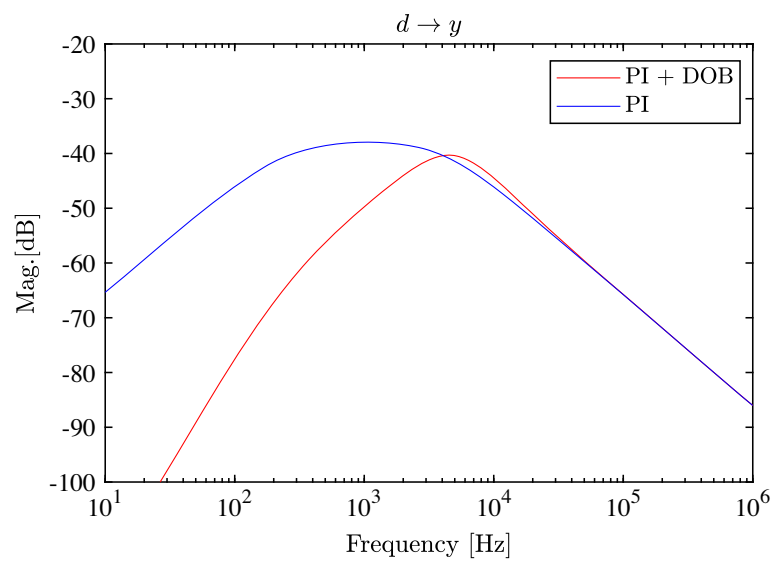

FIG. 16. Upper boundary for the singular values of the closedloop transfer functions from $d$ to $y$ in the cases of the two different control strategies.
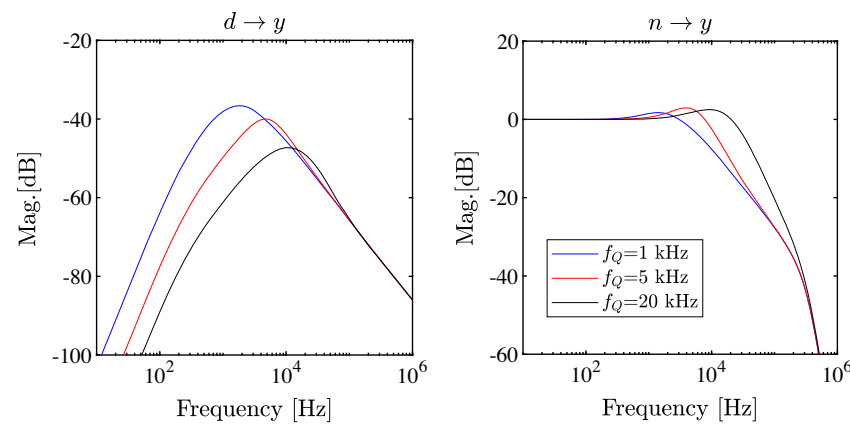

FIG. 17. Upper boundary for the singular values of the closedloop transfer function from $d$ and $n$ to $y$ in terms of different $f_{Q}$.

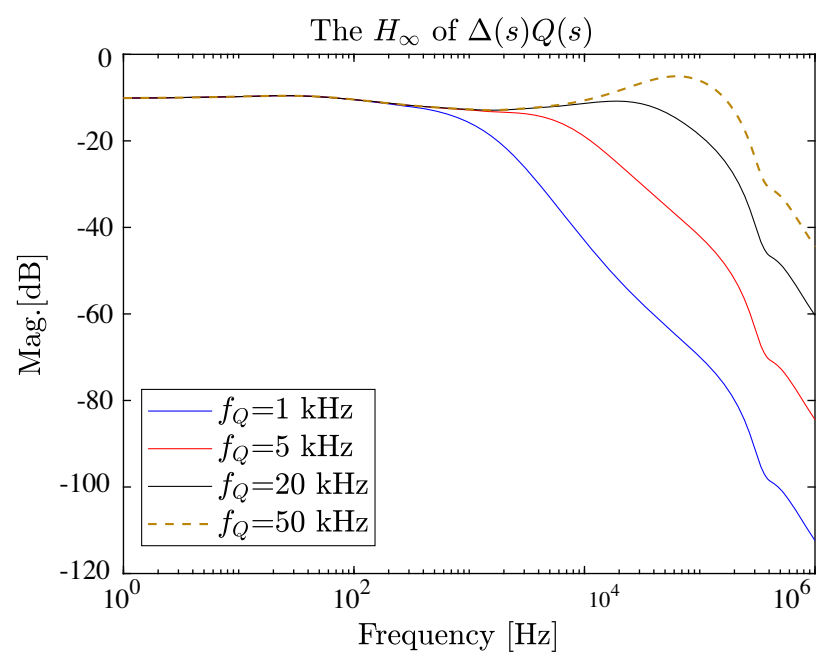

FIG. 18. $H_{\infty}$ norm of $\Delta(s) Q(s)$. The restrictions for the uncertainties are given in Table II.

Finally, we inspect the robust stability of the DOB control. The actual system $G_{p}$ is subjected to several uncertainties (e.g., cavity detuning, calibration error of loop phase). On the other hand, the mismatches always exist in the parameters of $G_{n}$. The relation between actual plant model and nominal model can be expressed by

$$
G_{p}(s)=G_{n}(s)[1+\Delta(s)]
$$

where the variable transfer function $\Delta(s)$ describes uncertainty of the plant. The sufficiency criterion of robust stability for DOB controller is given by

$$
\|\Delta(j \omega) Q(j \omega)\|_{\infty} \leq 1
$$

Figure 18 plots the $H_{\infty}$ in terms of different $f_{Q}$. Here, we consistently assume the restrictions of the uncertainties are listed in Table II. It can be seen that if the $f_{Q}$ is larger than $50 \mathrm{kHz}$, the DOB control is subject to a risk of unstable. In our experiment, we limited the $f_{Q}$ to be less than $20 \mathrm{kHz}$.

\section{EXPERIMENTAL VERIFICATION}

To validate DOB control in a pulsed rf system, we first installed this controller on ML1 and ML2 because these two cavities contribute the main part of the total beam energy. For cavities in the injector, we consider installing the controller in a future study. The cavity parameters of these two cavities are listed in Table I.

\section{A. Pulse-to-pulse drifts rejection}

When ML2 was operated at $V_{c}=10.5 \mathrm{MV}$, a pulse-topulse fluctuation was observed in the measured detuning. This fluctuation led to a pulse-to-pulse amplitude and phase drifts of the cavity voltage as shown in Fig. 6. However, 

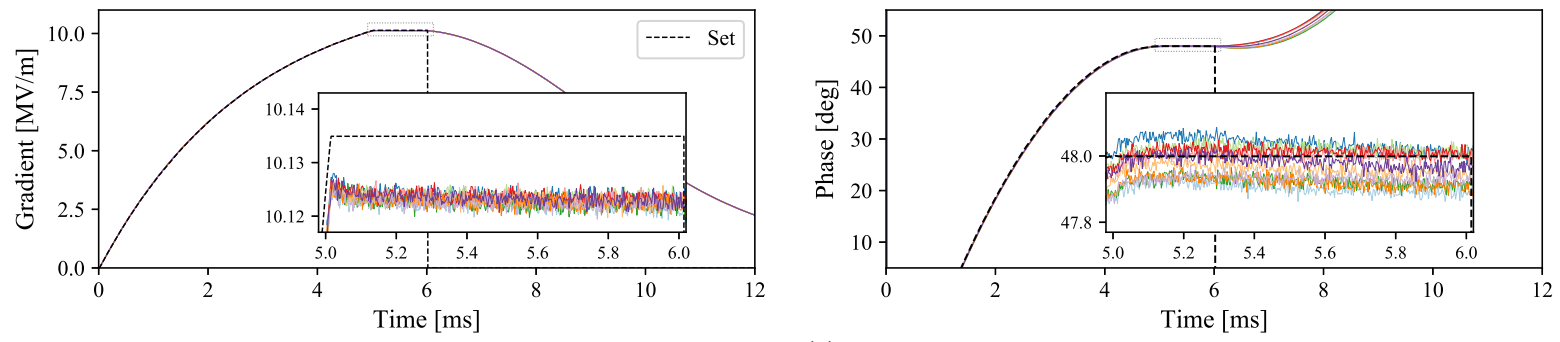

(a)
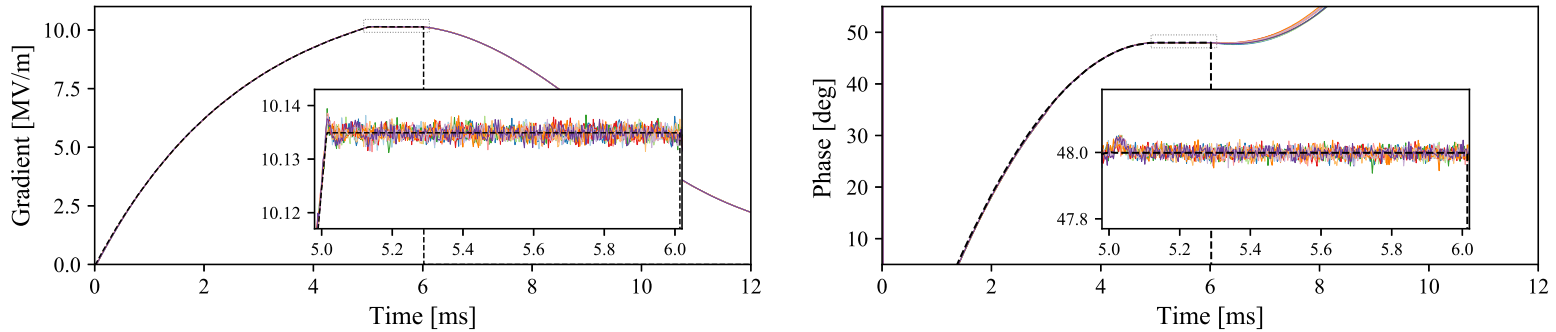

(b)

FIG. 19. Amplitude (left) and phase (right) stability in $\mathrm{ML2}\left(V_{c}=10.5 \mathrm{MV}\right)$ in the case of (a): $\mathrm{P}$ control and (b): $\mathrm{P}+\mathrm{DOB}$ control in the absence of the beam. This figure shows 10 randomly selected pulses from the data during one hour.

with the current PI FB control, it is not sufficient to reject these drifts. Figure 19(a) shows the amplitude and phase of 10 pulses in the case of $\mathrm{P}$ individual control $\left(K_{P}=50\right.$ and
$\left.K_{I}=0\right)$ in the absence of the beam. These 10 pulses are randomly selected from the saved pulse data during one hour. The pulse-to-pulse error is clearly observed in the
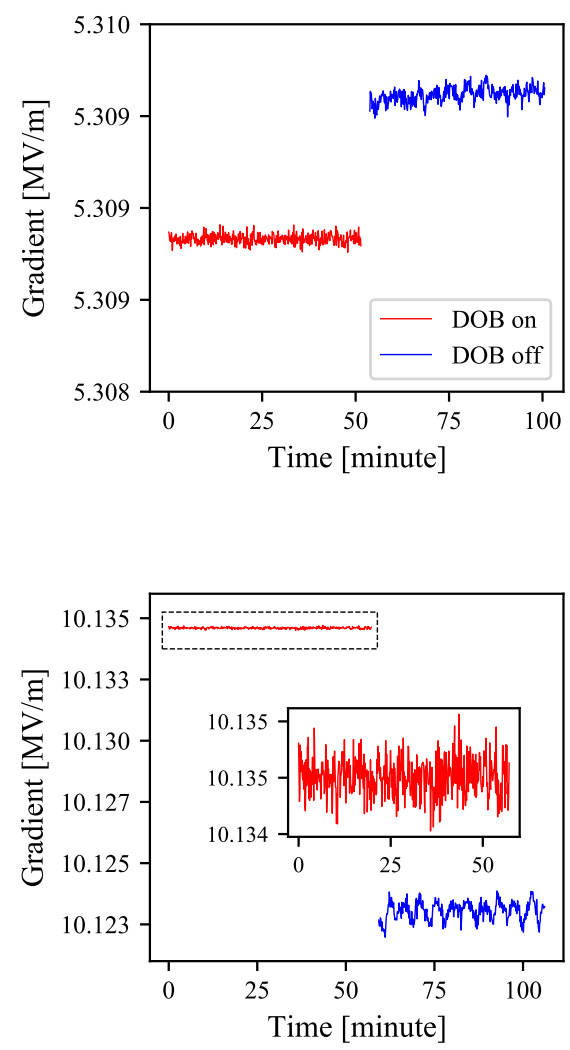

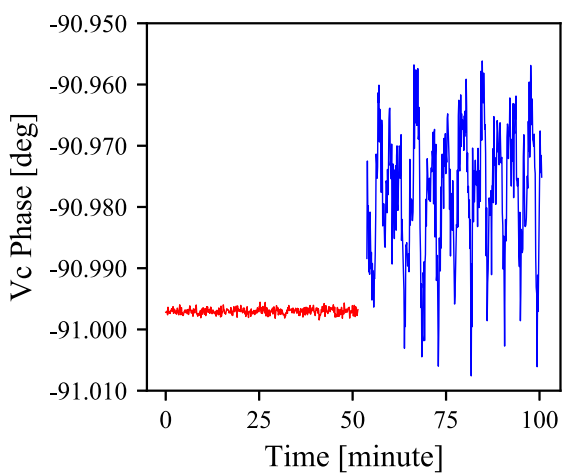

(a)

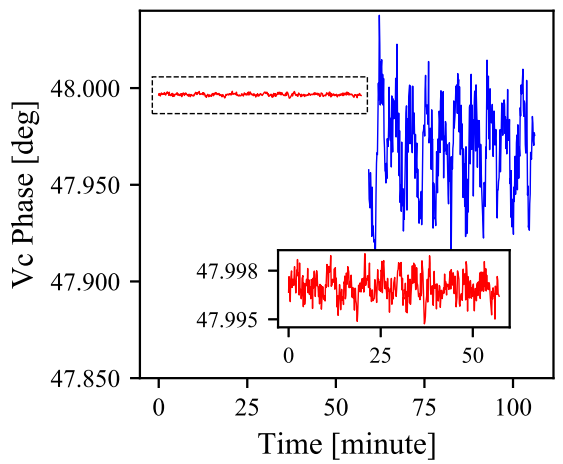

(b)
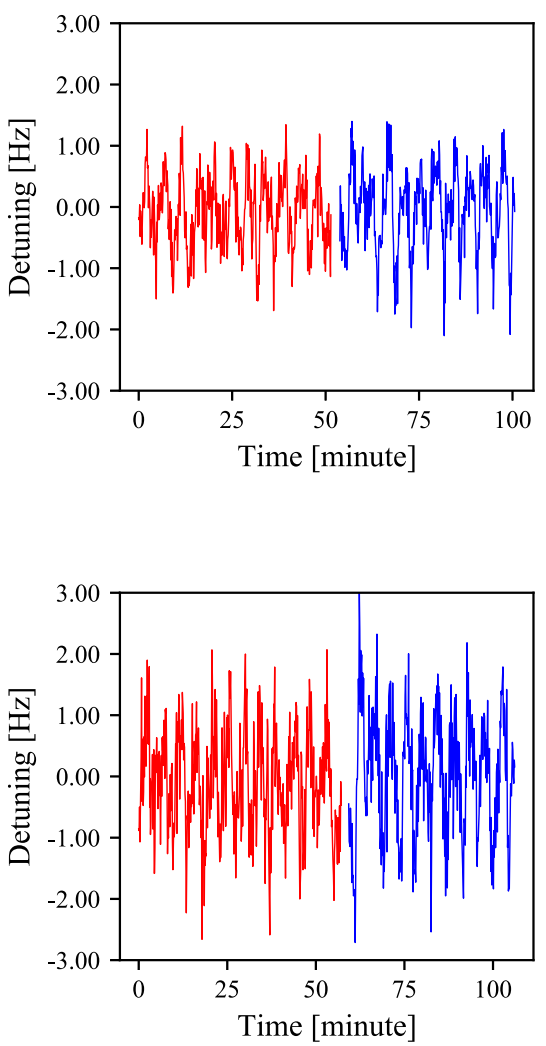

FIG. 20. Pulse to pulse stabilities of (a): ML1 ( $\left.V_{c}=5.5 \mathrm{MV}\right)$ and (b) ML2 ( $\left.V_{c}=10.5 \mathrm{MV}\right)$ in the case of P control (blue) and $\mathrm{P}+\mathrm{DOB}$ control (red) with $5 \mathrm{kHz} Q$ filter in the absence of the beam. 
TABLE III. Performance of DOB control for rejecting the pulse-to-pulse fluctuations in the absence of the beam.

\begin{tabular}{|c|c|c|c|c|c|c|c|c|c|}
\hline \multirow[b]{2}{*}{ Cavity } & \multirow[b]{2}{*}{$V_{c}[\mathrm{MV}]$} & \multicolumn{4}{|c|}{ Control method and LLRF parameters } & \multicolumn{2}{|c|}{ Intrapulse stability } & \multicolumn{2}{|c|}{ Pulse to pulse stability } \\
\hline & & Method & $\left(K_{P}, K_{I}\right)$ & $f_{Q}[\mathrm{kHz}]$ & Filter & $\Delta A / A[\% \mathrm{rms}]$ & $\Delta \phi\left[^{\circ} \mathrm{rms}\right]$ & $\Delta A / A[\% \mathrm{rms}]$ & $\Delta \phi\left[^{\circ} \mathrm{rms}\right]$ \\
\hline \multirow[t]{2}{*}{ ML1 } & 5.5 & $\mathrm{P}$ & $(80,0)$ & Not used & $250 \mathrm{kHz}$ IIR (4th) & 0.016 & 0.015 & 0.0007 & 0.0103 \\
\hline & & $\mathrm{P}+\mathrm{DOB}$ & & 5 & & 0.016 & 0.015 & 0.0005 & 0.0005 \\
\hline \multirow[t]{2}{*}{ ML2 } & 10.5 & $\mathrm{P}$ & $(50,0)$ & Not used & $250 \mathrm{kHz}$ IIR (4th) & 0.010 & 0.016 & 0.0040 & 0.0231 \\
\hline & & $\mathrm{P}+\mathrm{DOB}$ & & 5 & & 0.011 & 0.014 & 0.0003 & 0.0006 \\
\hline
\end{tabular}
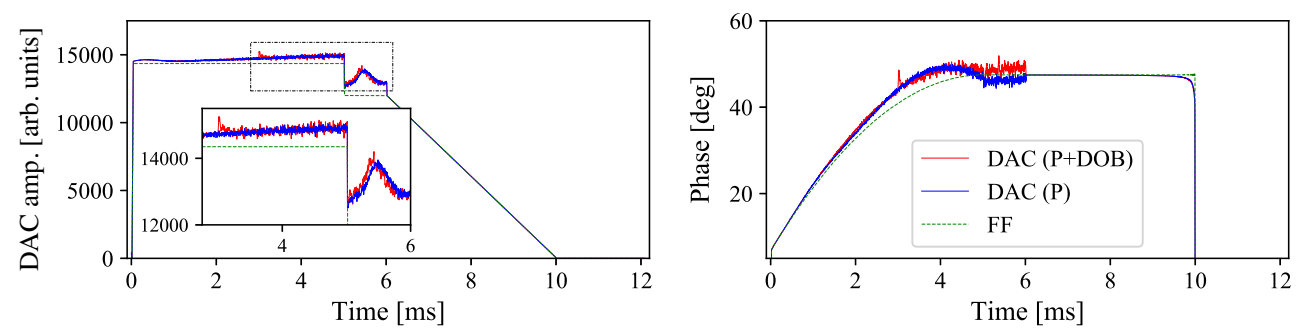

(a)
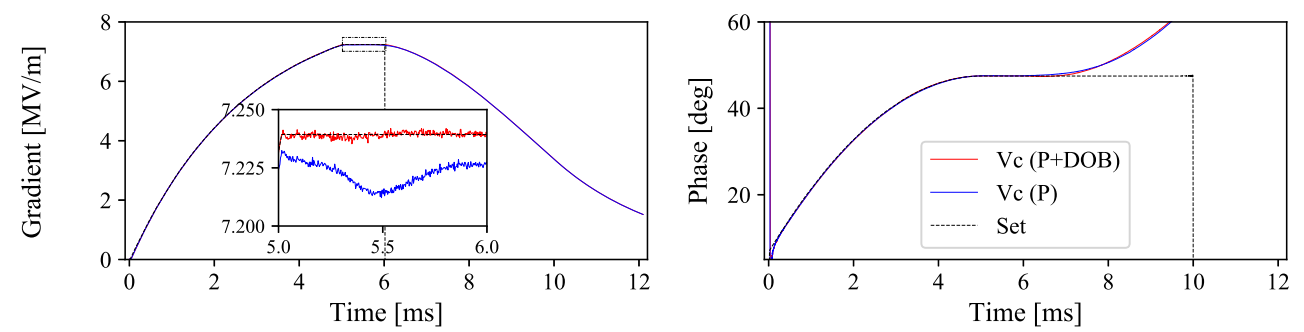

(b)

FIG. 21. Comparison of P control (blue) and P + DOB control (red) on ML2 operated at 10.5 MV. (a) Amplitude and phase of DAC output. (b) Amplitude and phase of cavity gradient. The DOB controller is activated after approximately 3 ms delay of the rf gate. The filling on resonance technique is applied in the design of the pulse pattern.
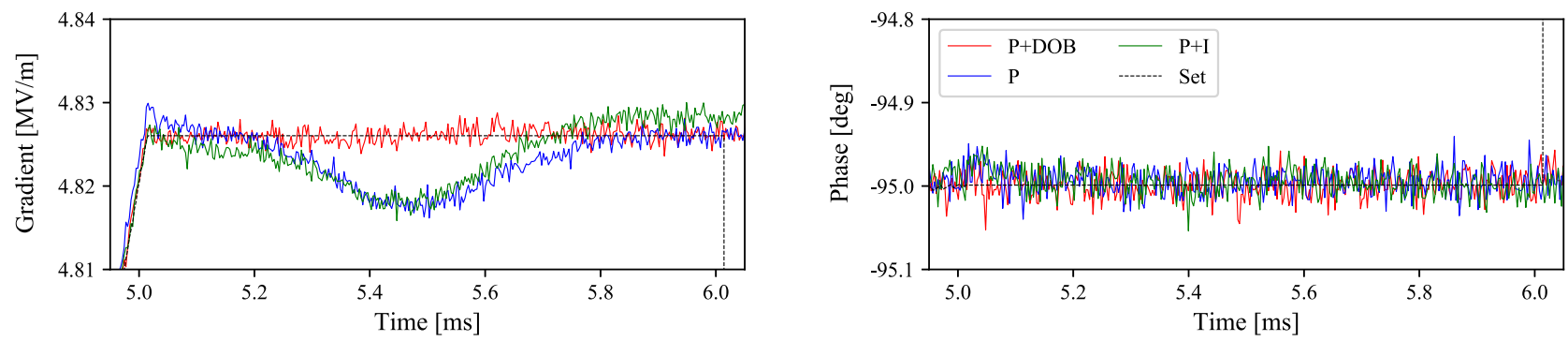

(a)
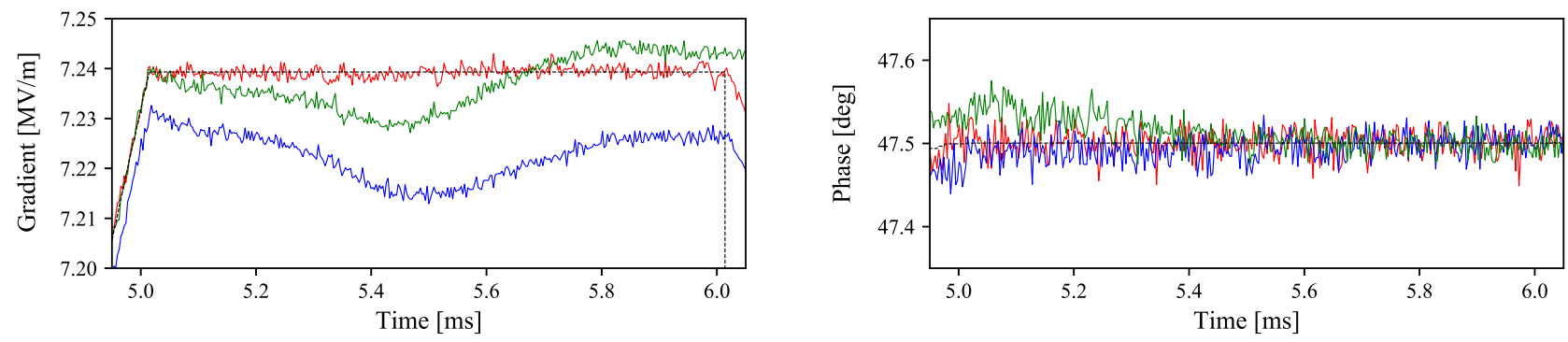

(b)

FIG. 22. P control (blue) versus PI control (green) versus P + DOB control (red) on ML1 and ML2 in the presence of long pulse beam. (a) Amplitude and phase stabilities during the flat-top of the pulse rf on ML1 at 5 MV. (b) Amplitude and phase stabilities during the flattop of the pulse rf on ML2 at 7.5 MV. The $f_{Q}$ in the DOB controller is selected to be $7.5 \mathrm{kHz}$ to reach a compromise. 
TABLE IV. Performance of DOB control in the beam-loading compensation during the cERL beam commissioning.

\begin{tabular}{|c|c|c|c|c|c|c|c|c|c|c|}
\hline \multirow[b]{2}{*}{ Cavity } & \multirow[b]{2}{*}{$V_{c}[\mathrm{MV}]$} & \multicolumn{4}{|c|}{ Control method and LLRF parameters } & \multicolumn{2}{|c|}{ Intrapulse stability } & \multicolumn{3}{|c|}{ Beam conditions } \\
\hline & & Method & $\left(K_{P}, K_{I}\right)$ & $f_{Q}[\mathrm{kHz}]$ & Filter & $\begin{array}{c}\Delta A / A \\
{[\% \mathrm{rms}]}\end{array}$ & $\begin{array}{c}\Delta \phi \\
{\left[{ }^{\circ} \mathrm{rms}\right]}\end{array}$ & $\begin{array}{c}I_{b} \\
{[\mu \mathrm{A}]}\end{array}$ & $\begin{array}{l}\text { Width } \\
{[\mathrm{ms}]}\end{array}$ & $\begin{array}{c}\phi_{b} \\
\text { [deg] }\end{array}$ \\
\hline \multirow[t]{3}{*}{ ML1 } & 5.5 & $\mathrm{P}$ & $(80,0)$ & Not used & $250 \mathrm{kHz}$ IIR (4th) & 0.066 & 0.015 & 66 & 0.7 & 0 \\
\hline & & PI & $\left(80,1.2 \times 10^{5}\right)$ & Not used & & 0.072 & 0.016 & & & \\
\hline & & $\mathrm{P}+\mathrm{DOB}$ & $(80,0)$ & 7.5 & & 0.019 & 0.015 & & & \\
\hline \multirow[t]{3}{*}{ ML2 } & 10.5 & $\mathrm{P}$ & $(50,0)$ & Not used & $250 \mathrm{kHz}$ IIR (4th) & 0.063 & 0.015 & & & \\
\hline & & PI & $\left(50,1.2 \times 10^{5}\right)$ & Not used & & 0.071 & 0.019 & & & \\
\hline & & $\mathrm{P}+\mathrm{DOB}$ & $(50,0)$ & 7.5 & & 0.015 & 0.015 & & & \\
\hline
\end{tabular}

rf phase. To further improve the performance of the pulseto-pulse rf stability, we used the same PI parameters and switched on the DOB controller. The bandwidth of the $Q$ filter $f_{Q}$ is set to $5 \mathrm{kHz}$. The pulse-to-pulse performance is improved as shown in Fig. 19(b).

Figure 20 shows the long-term stabilities of average amplitude, phase, and detuning values in the flat-top region for ML1 $\left(V_{c}=5.5 \mathrm{MV}\right)$ and ML2 $\left(V_{c}=10.5 \mathrm{MV}\right)$ under the $\mathrm{P}$ control and $\mathrm{P}+\mathrm{DOB}$ control. The pulse to pulse drifts for amplitude and phase were obviously improved by DOB control. The stabilities are summarized in Table III.

\section{B. Beam-loading compensation}

To validate the DOB method for beam-loading compensation, an LLRF experiment with a long-pulse beam was
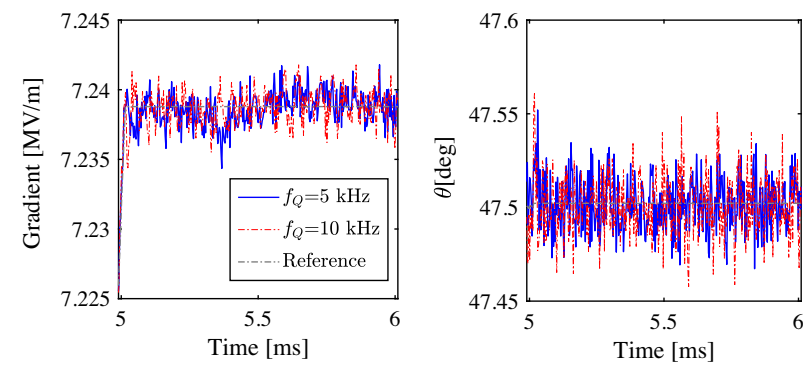

FIG. 23. Amplitude and phase stabilities during the flat top of the pulse rf in the case of $\mathrm{P}+\mathrm{DOB}$ control on ML2 at $7.5 \mathrm{MV}$ in terms of different $f_{Q}$.
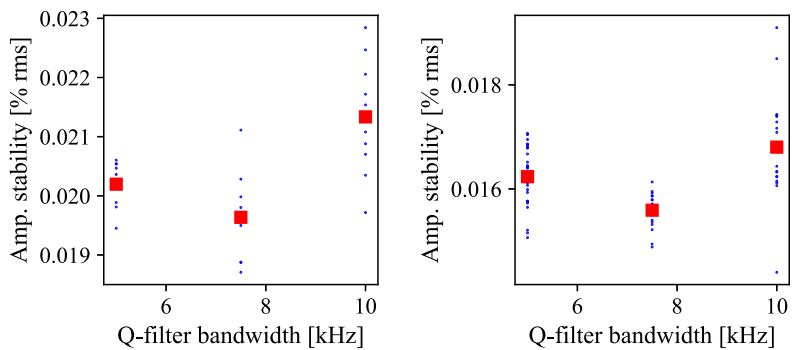

FIG. 24. $f_{Q}$-scanning result for ML1 $\left(V_{c}=5 \mathrm{MV}\right)$ and ML2 $\left(V_{c}=7.5 \mathrm{MV}\right) . f_{Q}=7.5 \mathrm{MV}$ is determined as the optimal bandwidth according to amplitude performance. performed in the cERL. The pulse width of the beam was approximately $0.7 \mathrm{~ms}$, and the peak beam current was approximately $0.07 \mathrm{~mA}$, as shown in Fig. 11(a).

Figure 21 shows the performance of the $\mathrm{P}$ control (blue) and $\mathrm{P}+\mathrm{DOB}$ control (red) on ML2. An FoR technique was applied in all three cases. In P control, very clear beamloading effects (approximately $0.2 \%$ peak to peak error) can be observed in the amplitude of the acceleration field. In the P + DOB case, the beam-loading effects in the amplitude are well compensated. The DOB controller is activated after an approximately $3 \mathrm{~ms}$ delay of the rf gate. A tiny increment of the DAC amplitude can be observed when activating the DOB control as shown in Fig. 21(a). During the flat-top stage from $5 \mathrm{~ms}$ to $6 \mathrm{~ms}$, a beamlike pulse is formed in the DAC output to compensate for beam loading. The $\mathrm{P}+\mathrm{DOB}$ control has a faster response compared to that of $\mathrm{P}$ control, which means the $\mathrm{P}+\mathrm{DOB}$ controller has a larger bandwidth with response to disturbance rejection.

Figure 22 shows the flat-top result for cavities in the ML in the case of P control, PI control, and P + DOB control. The main LLRF control parameters and corresponding rf stabilities in the presence of a long pulse beam are listed in Table IV. The parameter $\phi_{b}$ denotes the beam phase.

The selection $f_{Q}$ is a key issue in DOB control [9]. To evaluate the effect of $f_{Q}$, we measured system performance with different $f_{Q}$ values on ML2 at 7.5 MV. Figure 23 shows the rf amplitude (left) and phase (right) stabilities during the flat-top under $\mathrm{P}+\mathrm{DOB}$ control in the case of $f_{Q}=5 \mathrm{kHz}$ (blue) and $f_{Q}=10 \mathrm{kHz}$ (red). A tiny amplitude drop was still observed in the $f_{Q}=5 \mathrm{kHz}$ case. This amplitude drop disappeared after increasing the $f_{Q}$ to $10 \mathrm{kHz}$. However, as shown in both the amplitude and phase performances, the noise level is deteriorated in the case of $f_{Q}=10 \mathrm{kHz}$. This is consistent with the analytical study shown in Fig. 17. Figure 24 shows the $f_{Q}$ scanning result; we determine that $f_{Q}=7.5 \mathrm{kHz}$ achieves a trade-off between disturbance rejection and noise suppression.

\section{SUMMARY}

A new beamline to produce RIs was constructed in cERL at KEK. To increase the beam energy for RI manufacturing, the operation mode was switched from the original CW 
mode to the pulse mode. During beam commissioning in pulse mode, the pulse-to-pulse rf drifts caused by detuning fluctuation (and detuning offset) were observed. In addition, beam loading effects seriously influenced $\mathrm{rf}$ stability under current PI FB control.

The DOB control was applied for the pulsed mode operation. The pulse-to-pulse performance is improved by the DOB approach. Furthermore, obvious beam loading effects were well compensated.

The DOB method in this paper is designed for individual cavity control. Reference [9] used DOB control to reject phase ripples on the vector-sum of only two cavities. As a future study, the validity of the DOB approach needs to be demonstrated in the case of the vector-sum control of a larger number of cavities.

\section{ACKNOWLEDGMENTS}

This paper is based on results obtained from a New Energy and Industrial Technology Development Organization (NEDO) project "Development of advanced laser processing with intelligence based on high-brightness and high-efficiency laser technologies (TACMI project). We would like to thank all members of the cERL commissioning team for providing the stable beam operation. Further, we would like to thank Editage [24] for English language editing.

[1] M. Akemoto et al., Construction and commissioning of compact energy-recovery linac at KEK, Nucl. Instrum. Methods Phys. Res., Sect. A 877, 197 (2018).

[2] J. Akimitsu et al., Evaluation of the KEK project implementation plan, https://www.kek.jp/ja/About/Organization Overview/Assessment/Roadmap/KEK-PIP_Evaluation.pdf.

[3] Y. Morikawa et al., New industrial application beam-line for the cERL in KEK, in Proceedings of the 10th International Particle Accelerator Conference (IPAC2019), Melbourne, Australia (JACoW, Geneva, 2019), THPMP012, p. 3475.

[4] K.Tsuchiya et al., Development of the Tandem undulators for cERL-FEL (in Japanese), in Proceedings of the 16th Annual Meeting of Particle Accelerator Society of Japan (PASJ2019), Kyoto, Japan (Particle Accelerator Society of Japan, Kyoto, 2019), FRPI018, 2019, p. 1064.

[5] E. Kako et al., Degradation and recovery of cavity performance in compact-ERL injector cryomodule, in Proceedings of 18th International Conference on RF Superconductivity (SRF2017), Lanzhou, China (JACoW, Geneva, 2017), MOPB097, p. 289.

[6] H. Sakai, E. Cenni, K. Enami, T. Furuya, M. Sawamura, K. Shinoe, and K. Umemori, Field emission studies in vertical test and during cryomodule operation using precise $\mathrm{x}$-ray mapping system, Phys. Rev. Accel. Beams 22, 022002 (2019).

[7] H. Sakai et al., Long-term operation with beam and cavity performance degradation in compact-ERL main linac in KEK, in Proceedings of 29th Linear Accelerator Conference
(LINAC2018), Beijing, China (JACoW, Geneva, 2018), TUPO008, p. 695.

[8] F. Qiu et al., RF commissioning of the compact energy recovery linac superconducting cavities in pulse mode, Nucl. Instrum. Methods Phys. Res., Sect. A 985, 164660 (2021).

[9] F. Qiu, S. Michizono, T. Miura, T. Matsumoto, M. Omet, and S. B. Wibowo, Application of disturbance observerbased control in low-level radio frequency system in a compact energy recovery linac at KEK, Phys. Rev. Accel. Beams 18, 092801 (2015).

[10] F. Qiu, T. Miura, T. Matsumoto, S. Michizono, H. Katagiri, D. Arakawa, and H. Nakajima, Active cancellation of power supply ripple effects in continuous wave superconducting radio frequency cavities, Nucl. Instrum. Methods Phys. Res., Sect. A 955, 163280 (2020).

[11] K. Ohishi, Microprocessor controlled dc motor for load insensitive position servo system, IEEE Transactions on Industrial Electronics 34, 44 (1987).

[12] T. Umeno and Y. Hori, Robust speed control of dc servomotors using modern two degrees-of-freedom controller design, IEEE Transactions on Industrial Electronics 38, 363 (1991).

[13] J. Odagiri, Fully embedded Epics-based Control of Low Level RF system for SuperKEKB, in Proceedings of the 1st International Particle Accelerator Conference (IPAC2010), Kyoto, Japan (JACoW, Geneva, 2010), WEPEB003, p. 2686.

[14] T. Schilcher, Digital signal processing in RF applications, in Proceedings of CERN accelerator School on Digital Signal Processing, CAS2007, Sigtuna, Sweden, 2007 (CERN, Geneva, Switzerland, 2008), p. 249.

[15] T. Schilcher, Ph.D. Thesis, Universitat Hamburg, 1988.

[16] F. Qiu et al., Digital filters used for digital feedback system at cERL, in Proceedings of the 27th Linear Accelerator Conference (LINAC14), Geneva, Switzerland (JACoW, Geneva, Switzerland, 2014), MOPP074, p. 227.

[17] A. Brandt, Ph.D. thesis, Universitat Hamburg, 2007.

[18] M. U. Liepe, PhD thesis, University of Hamburg, 2001.

[19] B. W. Sigit, T. Matsumoto, S. Michizono, T. Miura, F. Qiu, and N. Liu, Digital low level rf control system for the International Linear Collider, Phys. Rev. Accel. Beams 21, 082004 (2018).

[20] V. Ayvazyan et al., Optimization of filling procedure for TESLA-type cavities for Klystron RF power minimization for European XFEL, in Proceedings of International Particle Accelerator Conference (IPAC2010), Kyoto, Japan (JACoW, Geneva, 2010), TUPEA039, p. 1416.

[21] K. Umemori et al., Long-term operation experience with beams in compact ERL cryomodules, in Proceedings of 18th International Conference on RF Superconductivity (SRF2017), Lanzhou, China (JACoW, Geneva, 2017), THYA06, p. 736.

[22] C. Schmidt, Ph.D. thesis, Technische Universitat HamburgHarburg, 2010.

[23] S. J. Mason, Feedback Theory: Further Properties of Signal Flow Graphs, Research Laboratory of Electronics (Massachusetts Institute of Technology, Cambridge, MA, 1956), pp. 920-926.

[24] www.editage.com. 